\title{
Preclinical cardiovascular abnormalities in patients in early stages of renal disease without nephrotic syndrome
}

Hypertension Research (2009) 32, 1155-1156; doi:10.1038/hr.2009.141; published online 23 October 2009

Cardiovascular morbidity and mortality are elevated in patients with chronic kidney disease (CKD). ${ }^{1}$ Most of the studies evaluating the correlations between chronic renal dysfunction and cardiovascular disease (CVD) have been performed in patients with endstage renal failure, whereas few data are available regarding cardiovascular abnormalities in patients with early-stage renal disease. ${ }^{2}$ Recently, Andoh et al. ${ }^{3}$ showed that, in nondiabetic patients with nephrotic syndrome (NS) in the early stages of CKD, there is an impairment of the circadian rhythm of blood pressure (BP), leading to a high percentage of non-dipper subjects, who have been reported to be at an increased risk of CVD. ${ }^{4}$ These alterations were significantly correlated with the degree of proteinuria and serum albumin levels. By contrast, the study did not find any significant differences in power spectral analysis of the heart rate between patients and healthy controls.

Here, we report the results of an observational study, which could be considered to be complementary to the study by Andoh et al. We evaluated the prevalence of inflammation and pre-clinical cardiovascular abnormalities in non-diabetic patients with primary glomerulonephritis, in the early phases of CKD without NS. We enrolled 41 subjects: 21 patients who had primary glomerulonephritis and were clinically normotensive (group GN) and 20 sex- and age-matched healthy control subjects (group C). The diagnosis of primary glomerulonephritis was based on renal biopsies. Only patients with a glomerular filtration rate $>60 \mathrm{ml} \mathrm{min}^{-1}$ were enrolled, corresponding to the KidneyDialysis Outcomes Quality Initiative stage I or II. ${ }^{5}$ Exclusion criteria were diabetes, obesity and NS. The histological diagnosis was immunoglobulin A nephropathy in 13 patients and focal segmental glomerulosclerosis in 8 patients. Clinical and cardiovascular evaluations were performed after the renal biopsy, before the beginning of any drug therapy. After the histological diagnosis, 14 patients underwent therapy with angiotensin I-converting enzyme inhibitors (ACEis), 5 with angiotensin II receptor blockers and 2 with ACEis associated with oral prednisone. The study protocol was approved by an independent ethics committee, and all subjects gave written informed consent.

Inflammation was assessed by measurement of fibrinogen plasma levels and highsensitivity C-reactive protein (hsCRP) serum levels by Cardiophase-hsCRP, Dade Behring (Newark, NJ, USA). Table 1 shows the characteristics of the two groups. There were no significant differences in age, gender, body mass index, cardiovascular risk factors and

creatinine clearance. The mean urinary protein excretion in group $\mathrm{GN}$ was $1.21 \pm 0.50 \mathrm{~g}$ every $24 \mathrm{~h}$. Levels of serum inflammatory markers were significantly higher in group GN than in group C: fibrinogen $359.9 \pm 59.4$ vs $239.4 \pm 35.5 \mathrm{mg} \mathrm{dl}^{-1}, \quad P<0.05$; hsCRP $4.62 \pm 4.26$ vs $1.08 \pm 0.64 \mathrm{mgl}^{-1}, \quad P<0.05$. Ambulatory BP was measured by $24-\mathrm{h}$ monitoring. We also evaluated the circadian rhythm of BP patterns to investigate the percentage of non-dipper subjects (that is, (nighttime $\mathrm{BP}$-daytime $\mathrm{BP}$ )/daytime $\mathrm{BP}>$ $-10 \%)$. Left ventricular (LV) mass indexed for body surface area (LVM/BSA) and systolic function (ejection fraction (EF)) were assessed by $2 \mathrm{D} / \mathrm{M}$-mode echocardiography. Endothelial function was investigated by quantification of flow-mediated dilation (FMD) of the brachial artery. FMD was evaluated by ultrasonographic imaging of the brachial artery at $1 \mathrm{~min}$ post-ischemia, that is,

Table 1 Characteristics of the patients

\begin{tabular}{|c|c|c|}
\hline & Glomerulonephritis & Controls \\
\hline$N$ & 21 & 20 \\
\hline Age (years) & $36.7 \pm 9.7$ & $31.4 \pm 5.9$ \\
\hline Gender (male/female) & $11 / 10$ & $11 / 9$ \\
\hline $\mathrm{BMI}\left(\mathrm{kg} \mathrm{m}^{-2}\right)$ & $24 \pm 4.2$ & $23.9 \pm 3.6$ \\
\hline Familiar cardiovascular history (\%) & $6(29 \%)$ & $6(30 \%)$ \\
\hline Current smokers (\%) & $5(21 \%)$ & $4(20 \%)$ \\
\hline Blood glucose $\left(\mathrm{mg} \mathrm{dl}^{-1}\right)$ & $91.5 \pm 8.4$ & $89.4 \pm 7.6$ \\
\hline Total cholesterol (mg dl-1) & $209.4 \pm 40.6$ & $164.25 \pm 12.4$ \\
\hline HDL-cholesterol (mg dl $\left.{ }^{-1}\right)$ & $56.1 \pm 16.0$ & $43.1 \pm 8.4$ \\
\hline Fibrinogen plasma levels (mg dl-1) & $359.9 \pm 59.4^{*}$ & $239.4 \pm 35.5$ \\
\hline hsCRP (mg I-1) & $4.62 \pm 4.26^{*}$ & $1.08 \pm 0.64$ \\
\hline Homocysteine plasma levels $\left(\mu \mathrm{mol} \mathrm{I}^{-1}\right.$ ) & $13.6 \pm 3.9$ & $13.1 \pm 1.7$ \\
\hline Creatinine clearance $\left(\mathrm{ml} \mathrm{min}{ }^{-1}\right)$ & $101.3 \pm 22.0$ & $104.4 \pm 9.4$ \\
\hline
\end{tabular}

Abbreviations: BMI, body mass index; HDL-cholesterol, high-density lipoprotein cholesterol; hsCRP: high-sensitivity C-reactive protein.

Data were expressed as mean \pm s.d. Comparisons between patients and controls were evaluated by Mann-Whitney test. ${ }^{*} P<0.05$ vs. control. 
Table 2 Cardiovascular evaluations

\begin{tabular}{llcc}
\hline Methods & Parameters & Glomerulonephritis & Controls \\
\hline & N & 21 & 20 \\
Echocardiography & LVM/BSA (g m $\left.{ }^{-2}\right)$ & $65.0 \pm 11.2$ & $75.8 \pm 17.4$ \\
& EF (\%) & $59.2 \pm 4.7$ & $60.9 \pm 4.6$ \\
Endothelial function assessment (FMD) & FMD (\% 1 min) & $12.9 \pm 7.2$ & $15.3 \pm 2.1$ \\
& FMD <10 (\%) & $7(33 \%)^{*}$ & 0 \\
Autonomic function assessment & SDANN24H (ms) & $105.3 \pm 26.9 * *$ & $147.7 \pm 22.6$ \\
& SDNN24H (ms) & $124.4 \pm 26.5^{*}$ & $158.6 \pm 23.6$ \\
(HRV-time domain) & RMSSD24H (ms) & $57.6 \pm 26.7$ & $43.4 \pm 10.3$ \\
Clinical BP & PNN50 (\%) & $15.9 \pm 8.5$ & $14.6 \pm 5.6$ \\
ABPM & Systolic BP (mm Hg) & $123.4 \pm 9.9$ & $118.7 \pm 12.0$ \\
& Diastolic BP (mm Hg) & $72.0 \pm 10.5$ & $77.9 \pm 5.6$ \\
& 24-h Systolic BP (mm Hg) & $120.4 \pm 5.3$ & $114.3 \pm 8.8$ \\
& 24-h Diastolic BP (mm Hg) & $77.4 \pm 8.7$ & $74.4 \pm 5.7$
\end{tabular}

Abbreviations: ABPM, 24h-ambulatory blood pressure monitoring; BP, blood pressure; EF, ejection fraction; FMD: flow-mediated dilation; HRV, heart rate variability; LVM/BSA, left ventricular mass indexed for body surface area; pNN50, percentage of NN intervals greater than $50 \mathrm{~ms}$; RMSSD, square root of the mean squared differences of successive NN intervals; SDANN, the s.d. of the average NN intervals calculated over short periods; SDNN, s.d. of the normal-to-normal QRS complex intervals. Data were expressed as mean \pm s.d. Comparisons between patients and controls were evaluated using Mann-Whitney test. Prevalence of endothelial dysfunction between groups was analyzed using Fisher's exact test. $P$ values $<0.05$ were considered to indicate statistical significance.

${ }^{*} P<0.05$

$* * P<0.01$ vs control

$100 \times\left(\right.$ Diameter $_{(1 \mathrm{~min})}-$ Diameter $\left._{(\text {basal })}\right) /$ Dia $\operatorname{meter}_{(\text {basal) }}$. Compared with the basal value, FMD $<10 \%$ was considered pathological. ${ }^{6}$ Finally, sympathetic balance was studied by evaluating the heart rate variability (HRV), which was calculated from 24-h electrocardiographic monitoring, by analyzing time domain measures. Each QRS complex was detected, and the normal-to-normal (NN) intervals (intervals between adjacent QRS complexes resulting from sinus node depolarization) were determined. Next, we calculated the s.d. of the NN intervals (SDNN), the s.d. of the average NN intervals (SDANN) calculated over short periods, the square root of the mean squared differences of successive $\mathrm{NN}$ intervals and the percentage of $\mathrm{NN}$ intervals $>50 \mathrm{~ms}^{7}$ Table 2 reports the results of the cardiovascular evaluations. LVM and EF showed differences between groups GN and $C$ that were not statistically significant. Both clinical and ambulatory BP evaluations showed values in the normal range that were not dissimilar between groups $\mathrm{GN}$ and $\mathrm{C}$ (average 24-h systolic BP $120.4 \pm 5.3 v s$. $114.3 \pm 8.8 \mathrm{~mm} \mathrm{Hg} ;$ average 24 -h diastolic BP $77.4 \pm 8.7$ vs. $74.4 \pm 5.7 \mathrm{~mm} \mathrm{Hg}$, respectively). There were no significant differences in the percentage of non-dippers between the two groups. Furthermore, compared with group C, group GN showed a significantly higher prevalence of pathological FMD (33 vs. $0 \%, P<0.05)$ and a lower percentage of post-ischemic vasodilation that did not reach statistical significance $(12.9 \pm 7.2$ vs. $15.3 \pm$ $2.1 \%)$. Lower values of HRV were also observed in GN, SDANN $(105.3 \pm 26.9 v s$. $147.7 \pm 22.6 \mathrm{~ms}, \quad P<0.01) \quad$ and $\quad$ SDNN $(124.4 \pm 26.5$ vs. $\quad 158.6 \pm 23.6 \mathrm{~ms}, \quad P<0.05)$, suggesting an impairment of autonomic function with a sympathetic overdrive. In this study, in agreement with previous reports, ${ }^{8}$ we demonstrated the presence of chronic inflammation in patients with primary glomerulonephritis in the early stages of CKD without NS, as shown by elevated levels of fibrinogen and hsCRP. In addition, we also showed that these patients presented an endothelial dysfunction and a sympathovagal imbalance when compared with healthy subjects, even if they were young and in good clinical condition, without hypertension or echocardiographically overt abnormalities of LV structure and systolic function. Coexistence of inflammation, endothelial dysfunction and autonomic dysfunction could indicate that these patients, although free from cardiovascular symptomatology, are at a high risk of developing atherosclerosis, independently of BP. ${ }^{9}$ Interestingly, our patients showed a different cardiovascular profile than those reported by Andoh et al., as we did not find an impairment of circadian BP rhythm, but we did detect significant alterations in sympathovagal activity. These findings could be explained by the different characteristics of the patients evaluated-in particular, the presence or absence of NS could have a crucial role. Thus, it can be assumed that patients with CKD, even in the early stages of renal disease, present different patterns of cardiovascular abnormalities and risk, depending on the underlying renal dis- order and its clinical expression. For these reasons, we suggest that evaluation of cardiovascular risk in CKD patients should be done on an individual basis, taking into consideration several factors, such as proteinuria, inflammatory markers, BP control, sympathetic activity, etc. However, larger and longitudinal studies are required to investigate the real clinical impact of the early pre-clinical alterations on long-term cardiovascular risk in CKD patients.

\section{CONFLICT OF INTEREST}

The authors declare no conflict of interest.

Pasquale Esposito ${ }^{1}$, Vittorio Palmieri ${ }^{2}$, Paola Migliaresi ${ }^{3}$, Salvatore Pezzullo ${ }^{3}$, Stefania Martino ${ }^{3}$ and Mario M Balletta ${ }^{4}$

${ }^{1}$ Department of Nephrology, Dialysis and Transplantation, University of Pavia and Fondazione IRCCS Policlinico San Matteo, Pavia, Italy; ${ }^{2}$ Unit of Cardiology, 'Ospedale dei Pellegrini', Naples, Italy; ${ }^{3}$ Department of Clinical and Experimental Medicine,

'Federico II' University of Naples, Naples, Italy and ${ }^{4}$ Department of Nephrology, 'Federico II' University of Naples, Naples, Italy

E-mail: pasqualeesposito@hotmail.com

1 Foley RN, Parfrey PS, Sarnak MJ. Clinical epidemiology of cardiovascular disease in chronic renal disease. Am J Kidney Dis 2008; 32: S112-S119.

2 Edwards NC, Hirth A, Ferro CJ, Townend JN, Steeds RP. Subclinical abnormalities of left ventricular myocardial deformation in early-stage chronic kidney disease: the precursor of uremic cardiomyopathy? J Am Soc Echocardiogr 2008; 21: 1293-1298.

3 Andoh D, Kobayashi M, Yasuda G, Hirawa N, Saka S, Yatsu K, Yamamoto Y, Umemura S. Loss of nocturnal decline of blood pressure in non-diabetic patients with nephrotic syndrome in the early and middle stages of chronic kidney disease. Hypertens Res 2009; 32: 364-368.

4 Giles TD. Circadian rhythm of blood pressure and the relation to cardiovascular events. J Hypertens Suppl 2006; 24: S11-S16.

5 National Kidney Foundation. K/DOQI clinical practice guidelines for chronic kidney disease: evaluation, classification, and stratification. Am J Kidney Dis 2002; 39 (Suppl 1): S1-S266.

6 Ghiadoni L, Versari D, Giannarelli C, Faita F, Taddei S. Non-invasive diagnostic tools for investigating endothelial dysfunction. Curr Pharm Des 2008; 14: 3715-3722.

7 Task Force of the European Society of Cardiology and the North American Society of Pacing and Electrophysiology. Heart rate variability: standards of measurement, physiological interpretation and clinical use.. Circulation 1996; 93: 1043-1065.

8 Nelson CL, Karschimkus CS, Dragicevic G, Packham DK, Wilson AM, O'Neal D, Becker GJ, Best JD, Jenkins AJ. Systemic and vascular inflammation is elevated in early IgA and type 1 diabetic nephropathies and relates to vascular disease risk factors and renal function. Nephrol Dial Transplant 2005; 20: 2420-2426.

9 Stam F, van Guldener C, Becker A, Dekker JM, Heine RJ, Bouter LM, Stehouwer CD. Endothelial dysfunction contributes to renal function-associated cardiovascular mortality in a population with mild renal insufficiency: the Hoorn study. J Am Soc Nephrol 2006; 17: 537-545. 